\title{
Gold-platinum alloy nanoparticles through water-in-oil microemulsion
}

\author{
Angshuman Pal
}

Received: 29 August 2014/ Accepted: 27 October 2014/Published online: 10 December 2014

(C) The Author(s) 2014. This article is published with open access at Springerlink.com

\begin{abstract}
Au}-\mathrm{Pt}$ alloy nanoparticles have been synthesized by the reduction of tetrachloroauric acid $\left(\mathrm{HAuCl}_{4}\right)$ and chloroplatinic acid $\left(\mathrm{H}_{2} \mathrm{PtCl}_{6}\right)$ using sodium borohydride in water-in-oil (w/o) microemulsion of water/Triton $\mathrm{X}-100 /$ cyclohexene/1-hexanol at $25 \pm 2{ }^{\circ} \mathrm{C}$. The observed nanoparticles looked like a chain with diameter of $2.5 \mathrm{~nm}$. The particles formation was characterized by UV-visible spectrometry and transmission electron microscopy images. The particles are crystalline confirmed by high-resolution transmission electron microscopy and diffraction pattern. A bright-field and dark-field images were compared to find out the nature of the nanochains.
\end{abstract}

Keywords Nanoparticles $\cdot$ Alloy $\cdot \mathrm{Au}-\mathrm{Pt}$

\section{Introduction}

Metallic or bimetallic nanocrystals of various shapes with tunable optical and electronic properties are valuable in the fabrication of a new generation electronics, sensing and photonic device [1-4]. Many methods based on a template-assisted system, where the template may be hard such as tube [5-7], wires [8-10], and soft such as surfactant [11-13], polymer [14, 15], biomacromolecule including DNA [16, 17] and viruses [18], are reported for the formation of metal nanorods or nanowires. Recently, Xie et al. [19] have described template-

Electronic supplementary material The online version of this article (doi:10.1007/s40097-014-0136-8) contains supplementary material, which is available to authorized users.

A. Pal $(\bowtie)$

Chemistry and Biomolecular Science, Clarkson University,

Potsdam, NY 13699, USA

e-mail: apal@clarkson.edu free method to the formation of metal nanowires. Preparation of metallic nanoparticles using microemulsion has also been well reported where ionic and nonionic surfactants are used. There are several reports available for bimetallic alloy particles, specially, synthesis of Au-Pt nanoparticles in microemulsion including computer simulation [20-23]. Microemulsions are colloidal "nano-dispersions" of water-inoil or oil-in-water, stabilized by a surfactant film. In water-inoil microemulsion, the size of the water droplet can be tuned by changing only one parameter such as water-to-surfactant molar ratio $\left(W_{0}\right)$. The size of nanoparticles is controlled by the size of the droplet of the microemulsion. In our previous report [24], we have described the advantages of the reverse micelle system for the preparation of $\mathrm{Au}-\mathrm{Ag}$ alloy nanoparticles.

In template-based method, soft templates are commonly used in the preparation of metallic and bimetallic nanomaterials. The tuning of crystal growth into a desirable geometrical form is important in the anisotropic growth of the particles in the soft template [19]. However, the method suffers from a disadvantage of tedious procedures for the preparation of template-free nanoparticles for further application. The present communication is a part of our ongoing efforts towards this direction to overcome the limitation.

Here we describe the synthesis of bimetallic Au-Pt nanocrystals within few seconds at room temperature using waterin-oil microemulsion (w/o). A systematic study of synthesis and characterization of $\mathrm{Au}-\mathrm{Pt}$ nanoparticles is undertaken.

\section{Materials and methods}

Materials

Tetrachloroauric acid $\left(\mathrm{HAuCl}_{4}, 99.9 \%\right.$ metal), chloroplatinic acid $\left(\mathrm{H}_{2} \mathrm{PtCl}_{6}, 8 \% \mathrm{wt}\right.$ aqueous solution), sodium 
borohydride (granular $99.99 \%$ metal basis) and 1-hexanol were purchased from Aldrich, Steinheim, Germany. Triton $\mathrm{X}-100$ and cyclohexane were purchased from SigmaAldrich, Steinheim, Germany.

Synthesis of gold-platinum bimetallic nanoparticles

The microemulsion system was composed of Triton X-100, cyclohexane, water, and 1-hexanol. The amounts of each component used in a typical reaction for $W_{\mathrm{o}}$ of 3 are $8 \%$ Triton X-100, water, and 1-hexanol at $0.027 \%(\mathrm{v} / \mathrm{v})$ each with cyclohexane as the remainder. The reactions were carried out at water-to-surfactant molar ratio 3 with metal ions concentration $2.7 \times 10^{-4} \mathrm{M}$ and reducing agent (sodium borohydride) concentration $1.4 \times 10^{-3} \mathrm{M}$. When w/o microemulsion systems containing a reducing agent and appropriate metal salt were mixed under constant stirring, instantaneous formation of the particles was observed.

\section{Measurement}

Size, shape, and particle size distributions were determined using a JEOL JEM-2011 transmission electron microscope operated at an accelerating voltage of $200 \mathrm{kV}$. Images were recorded using a Gatan DualVision 600t CCD camera attached to the microscope and were analyzed using Gatan Digital Micrograph Version 3.11.1. Samples were prepared for TEM analysis by placing a drop of the solution on a carbon-coated copper grid and drying in air. Visible spectra were recorded on a Perkin-Elmer Lambda 35 UV-Vis spectrophotometer.

\section{Results and discussion}

Gold-platinum bimetallic nanoparticles were prepared by reducing metal compounds in w/o microemulsion using sodium borohydride as a reducing agent. To optimize the particle size and stability of the microemulsion, $W_{0}$ was varied from 1 to 7 . With the increase in $W_{0}$, concentration of particles formed increases but stability of the microemulsion decreases. At $W_{0}=5$ and 7, particles were stable only for $1-2 \mathrm{~h}$ and were precipitated out thereafter. At $W_{0}<3$, microemulsion was stable, but particles concentration is very low. Hence, further study was carried out at $W_{0}=3$, where optimum concentration of metallic and bimetallic stable particles was obtained. The color change caused by the reduction of metal salts was found to be dependent upon the concentrations of $\mathrm{HAuCl}_{4}$ and $\mathrm{H}_{2} \mathrm{PtCl}_{6}$ in solution.

The visible spectrum (Fig. 1) shows the presence of an absorption band at $545 \mathrm{~nm}$, a characteristic surface

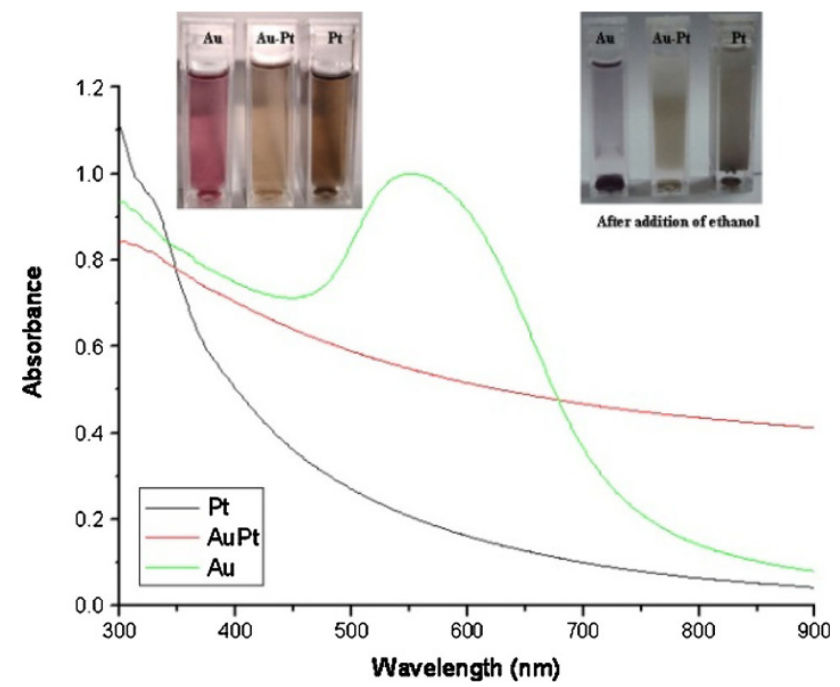

Fig. 1 UV-visible spectra of $\mathrm{Au}, \mathrm{Pt}$, and $\mathrm{Au}-\mathrm{Pt}$ nanoparticles. The inset shows the digital photographs of these three solutions before and after addition of ethanol

plasmon band for gold nanoparticles. Whereas for $\mathrm{Pt}$ nanoparticles, no characteristic absorption band is observed (Fig. 1) but a typical scattering pattern of Pt nanoparticles is exhibited. The disappearance of the surface plasmon absorption band at $545 \mathrm{~nm}$ in the visible spectrum (Fig. 1) indicates the formation of $\mathrm{Au}-\mathrm{Pt}$ bimetallic nanoparticles. This w/o microemulsion is thermodynamically stable system and it is stable for more than 6 months without showing any precipitation or optical changes. Any shortchain alcohol like ethanol and acetone can destabilize this microemulsion to get the desired product from this surfactant-based template. The digital photographs (inset, Fig. 1) show the change in color of three different nanoparticle solutions and the separation of particles after addition of ethanol to these solutions. The nanoparticles separated due to the destabilization of the microemulsion. The separated particles are centrifuged and washed repeatedly with ethanol and again centrifuged to get template-free product.

Figure $2 \mathrm{a}, \mathrm{b}$ shows the transmission electron microscope (TEM) images of 2-3 nm diameter sized gold and platinum nanoparticles, respectively. Insets show the high-resolution transmission electron microscope (HRTEM) image for $\mathrm{Au}$ [Fig. 2a] and Pt [Fig. 2b] nanoparticles which exhibits the crystalline nature of these particles. The electron diffraction pattern of $\mathrm{Au}$ nanoparticles [Fig S1] also confirms the crystalline character. The "d"spacing for $\mathrm{Au}$ and $\mathrm{Pt}$ nanocrystals was observed to be 0.235 and $0.227 \mathrm{~nm}$, respectively, which exactly coincides with $\mathrm{Au} \mathrm{d}(111)$ and cubic $\mathrm{Pt}$ d(111). Energy-dispersive X-ray spectroscopy (EDX) confirms the presence of Au [Fig S2] and Pt [Fig S3]. Figure 3 represents the $\mathrm{Au}-\mathrm{Pt}$ bimetallic particles of a 

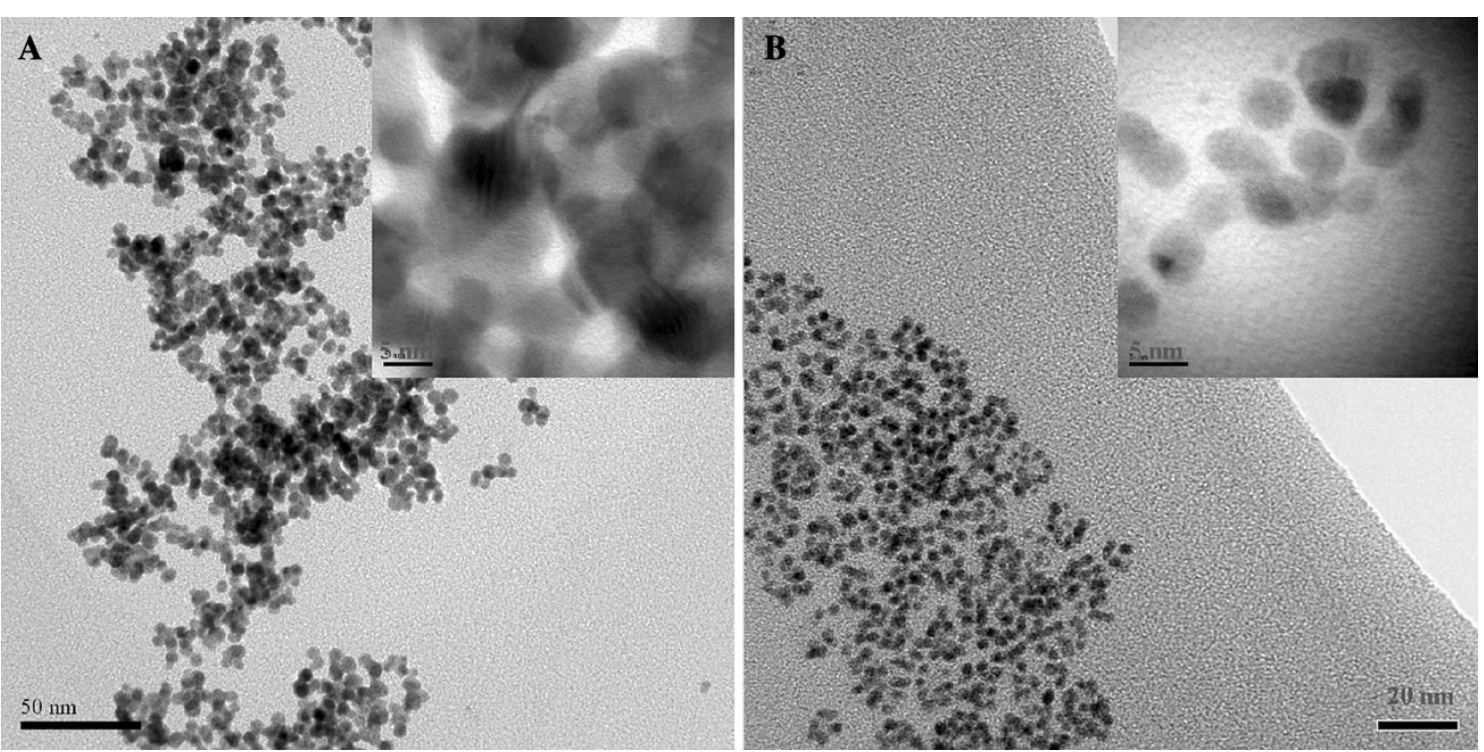

Fig. 2 TEM images of gold and platinum nanoparticles (insets are high magnification images of those particles)

Fig. 3 TEM (a) and HRTEM (b) images of $\mathrm{Au}-\mathrm{Pt}$ chain. c, d are the enlarge sections of (b)

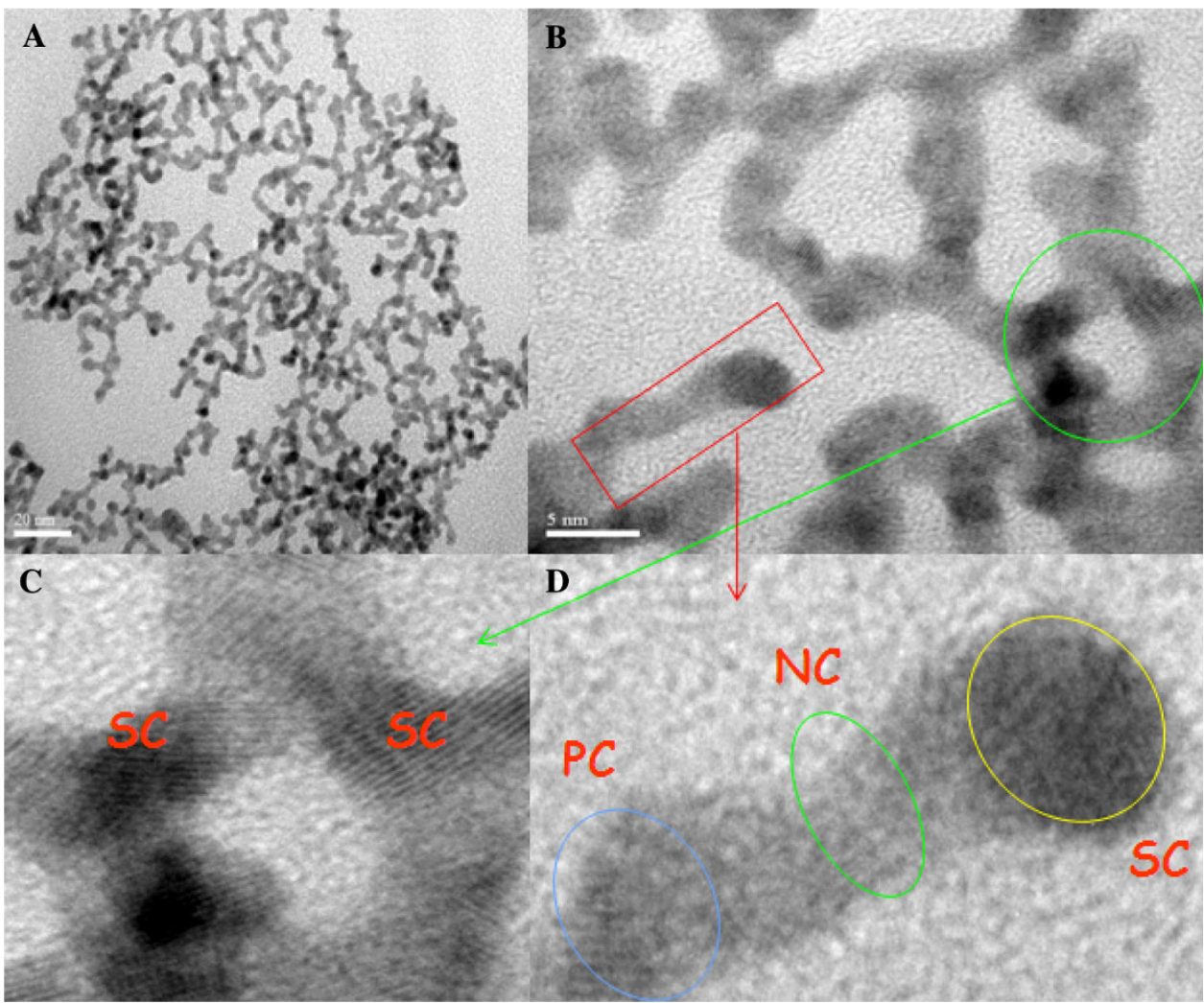

diameter $\sim 2.5 \mathrm{~nm}$. The nature of bimetallic nanoparticles is quite different from the corresponding monometallic particles. The particles are grown in close contact with each other, forming branched nanochain. The HRTEM image in Fig. $3 b$ shows the changes in the orientation of the lattice fringes, and randomly distributed crystalline and non-crystalline sections along the length of the extended chain indicating that the $\mathrm{Au}-\mathrm{Pt}$ nanoparticles are neither crystalline nor completely amorphous. Figure $3 \mathrm{c}$, d shows the two enlarge areas of Fig. 3b. These enlarge images shows the three different sections like single-crystalline $(S C)$, poly-crystalline $(P C)$, and non- 

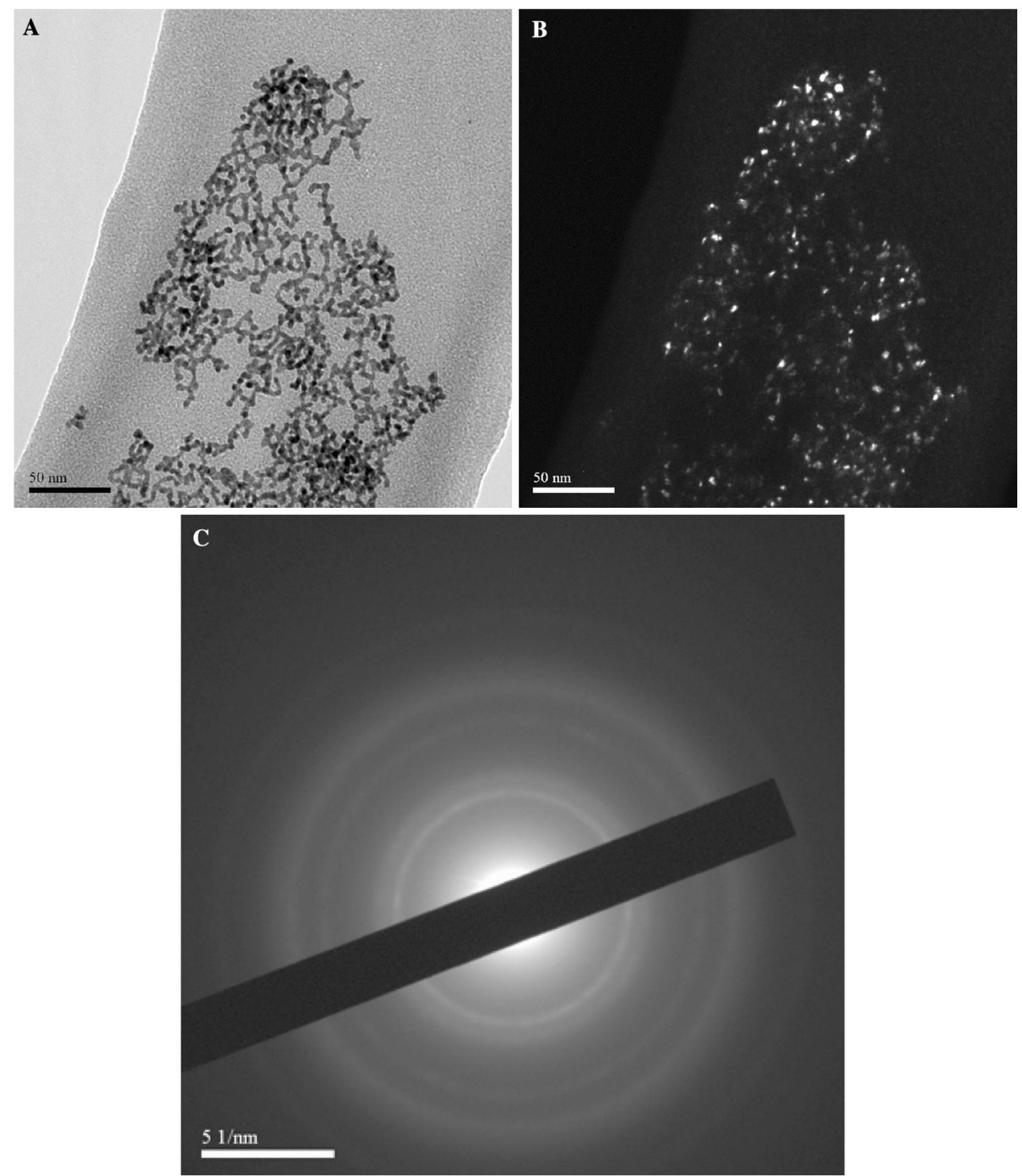

Fig. 4 Bright-field (a) and dark-field (b) images and electron diffraction (c) of Au-Pt nanoparticles

crystalline $(N C)$ along the length of the extended chain. In $N C$ and $P C$, section's orientation of the lattice fringes are absent. To verify the nature of this chain, a comparison of bright-field and dark-field images of a particular section of this Au-Pt chain is given in Fig. 4a, b, respectively. Randomly distributed bright spots in the dark-field image (Fig. 4b) indicate the crystalline part of this chain. Discontinuity of brighter spots on the darkfield image indicates the existence of crystalline and amorphous structures in this chain. The electron diffraction pattern and EDX for $\mathrm{Au}-\mathrm{Pt}$ chain is shown in Fig. 4c and Fig S4, respectively.

\section{Conclusions}

In summary, chains of gold-platinum nanoparticles with $2.5 \mathrm{~nm}$ diameter had been synthesized by the simultaneous 
reduction of $\mathrm{HAuCl}_{4}$ and $\mathrm{H}_{2} \mathrm{PtCl}_{6}$ using sodium borohydride in water-in-oil microemulsion at room temperature $\left(25 \pm 2{ }^{\circ} \mathrm{C}\right)$. The desired particles were separated from the template just by addition of ethanol. The extension of synthesis procedure and separation technique to other noble metallic and bimetallic nanoparticles can prove a landmark in the synthesis of nanomaterials.

Acknowledgments The authors are thankful to GUJCOST (Gandhinagar, Gujarat, India) for the financial support.

Open Access This article is distributed under the terms of the Creative Commons Attribution License which permits any use, distribution, and reproduction in any medium, provided the original author(s) and the source are credited.

\section{References}

1. Xia, Y., Yang, P., Sun, Y., Wu, Y., Mayers, B., Gates, B., Yin, Y., Kim, F., Yan, H.: One dimensional nanostructures: synthesis, characterization, and applications. AdV. Mater. (2003). doi:10. 1002/adma.200390087

2. Tang, Z., Kotov, N.A.: One-dimensional assemblies of nanoparticles: preparation, properties, and promise. Adv. Mater. (2005). doi:10.1002/adma.200401593

3. Liz-Marzan, L.M.: Tailoring surface plasmons through the morphology and assembly of metal nanoparticles. Langmuir (2006). doi: $10.1021 / \mathrm{la} 0513353$

4. Eustis, S., El-Sayed, M.A.: Why gold nanoparticles are more precious than pretty gold: noble metal surface plasmon resonance and its enhancement of the radiative and nonradiative properties of nanocrystals of different shapes. Chem. Soc. Rev. (2006). doi:10.1039/B514191E

5. Ajayan, P.M., Iijima, S.: Capillarity-induced filling of carbon nanotubes. Nature (1993). doi:10.1038/361333a0

6. Zhang, Y., Dai, H.: Formation of metal nanowires on suspended single-walled carbon nanotubes. Appl. Phys. Lett. (2000). doi:10. $1063 / 1.1324731$

7. Guan, L., Shi, Z., Li, H., You, L., Gu, Z.: Super-long continuous Ni nanowires encapsulated in carbon nanotubes. Chem. Commun. (2004). doi:10.1039/B405444J

8. Yin, Y., Lu, Y., Sun, Y., Xia, Y.: Silver nanowires can be directly coated with amorphous silica to generate well-controlled coaxial nanocables of silver/silica. Nano Lett. (2002). doi:10.1021/ nl025508+
9. Mayya, K.S., Gittins, D.I., Dibaj, A.M., Caruso, F.: Nanotubes prepared by templating sacrificial nickel nanorods. Nano Lett. (2001). doi:10.1021/nl015622c

10. Sun, X., Li, Y.: Cylindrical silver nanowires: preparation, structure, and optical properties. Adv. Mater. 17, 2626-2630 (2005). doi:10.1002/adma.200500957

11. Kim, F., Song, J.H., Yang, P.: Photochemical synthesis of gold nanorods. J. Am. Chem. Soc. (2002). doi:10.1021/ja028110o

12. Nikoobakht, B., El-Sayed, M.A.: Preparation and growth mechanism of gold nanorods (NRs) using seed-mediated growth method. Chem. Mater. (2003). doi:10.1021/cm0207321

13. Murphy, C.J., Gole, A.M., Hunyadi, S.E., Orendorff, C.J.: Onedimensional colloidal gold and silver nanostructures. Inorg. Chem. (2006). doi:10.1021/ic0519382

14. Zhang, D., Qi, L., Ma, J., Cheng, H.: Formation of silver nanowires in aqueous solutions of a double-hydrophilic block copolymer. Chem. Mater. (2001). doi:10.1021/cm0105007

15. Cornelissen, J.J.L.M., Heerbeek, R., Kamer, P.C.J., Reek, J.N.H., Sommerdijk, N.A.J.M., Nolte, R.J.M.: Silver nanoarrays templated by block copolymers of carbosilane dendrimers and polyisocyanopeptides. Adv. Mater. 14, 489-492 (2002)

16. Keren, K., Krueger, M., Gilad, R., Ben-Yoseph, G., Sivan, U., Braun, E.: Sequence-specific molecular lithography on single DNA molecules. Science (2002). doi:10.1126/science.1071247

17. Patolsky, F., Weizmann, Y., Lioubashevski, O., Willner, I.: Aunanoparticle nanowires based on DNA and polylysine templates. Angew. Chem. Int. Ed. 41, 2323-2327 (2002)

18. Knez, M., Bittner, A.M., Boes, F., Wege, C., Jeske, H., Maiss, E., Kern, K.: Biotemplate synthesis of 3-nm nickel and cobalt nanowires. Nano Lett. (2003). doi:10.1021/n10342545

19. Xie, J., Zhang, Q., Lee, J.Y., Wang, D.C.: General method for extended metal nanowire synthesis: ethanol induced self-assembly. J. Phys. Chem. C (2007). doi:10.1021/jp0768120

20. Hernández-Fernández, $P$., et al.: influence of the preparation route of bimetallic Pt-Au nanoparticle electrocatalysts for the oxygen reduction reaction. J. Phys. Chem. C (2007). doi:10.1021/ jp066812k

21. Wu, M., Chen, D., Huang, T.: Preparation of Au/Pt bimetallic nanoparticles in water-in-oil microemulsions. Chem. Mater. (2001). doi:10.1021/cm0006502

22. Habrioux, A., et al.: Structural and electrochemical studies of Au-Pt nanoalloys. Phys. Chem. Chem. Phys. (2009). doi:10. 1039/B820668F

23. Tojo, C., et al.: Cage-like effect in Au-Pt nanoparticle synthesis in microemulsions: a simulation study. Phys. Chem. Chem. Phys. (2014). doi:10.1039/C4CP02936D

24. Pal, A., Shah, S., Devi, S.: Preparation of silver, gold and silvergold bimetallic nanoparticles in w/o microemulsion containing TritonX-100. Colloid Surf A (2007). doi:10.1016/j.colsurfa.2007. 03.032 